\title{
Papulopustular Rash, CTCAE
}

National Cancer Institute

\section{Source}

National Cancer Institute. Papulopustular Rash, CT CAE. NCI Thesaurus. Code C143735.

A disorder characterized by an eruption consisting of papules (a small, raised pimple) and pustules (a small pus filled blister), typically appearing in face, scalp, and upper chest and back. Unlike acne, this rash does not present with whiteheads or blackheads, and can be symptomatic, with itchy or tender lesions. 